\title{
Thermoelectric properties of atomically thin silicene and germanene nanostructures
}

\author{
K. Yang, ${ }^{1}$ S. Cahangirov, ${ }^{1}$ A. Cantarero, ${ }^{2}$ A. Rubio, ${ }^{1}$ and R. D' Agosta ${ }^{1,3, *}$ \\ ${ }^{1}$ Nano-Bio Spectroscopy Group and ETSF Scientific Development Center, Departamento de Fisica de Materiales, \\ Universidad del Pais Vasco UPVIEHU, Avenida Tolosa 72, E-20018 San Sebastian, Spain \\ ${ }^{2}$ Instituto de Ciencia de Materiales, Universidad de Valencia, E-46071 Valencia, Spain \\ ${ }^{3}$ IKERBASQUE, Basque Foundation for Science, E-48011 Bilbao, Spain
}

(Received 3 October 2013; published 4 March 2014)

\begin{abstract}
The thermoelectric properties in one- and two-dimensional silicon and germanium structures have been investigated using first-principles density functional techniques and linear response for the thermal and electrical transport. We have considered here the two-dimensional silicene and germanene, together with nanoribbons of different widths. For the nano ribbons, we have also investigated the possibility of nano structuring these systems by mixing silicon and germanium. We found that the figure of merit at room temperature of these systems is remarkably high, up to 2.5 .
\end{abstract}

DOI: 10.1103/PhysRevB.89.125403

PACS number(s): 73.50.Lw, 63.22.-m

\section{INTRODUCTION}

Thermoelectric energy conversion is the ability of a device to convert a steady temperature gradient into an electrical current, and was first discovered by Seebeck in 1821 [1-3]. In a reverse mode operation, a thermoelectric device can be used as a cooler by maintaining a steady current in the device (Peltier effect) [1-3]. Recently, the quest for a highly efficient thermoelectric device has attracted tremendous interest due to significant potential industrial applications [1-5]. The efficiency of the thermoelectric conversion is characterized by a dimensionless parameter, called figure of merit

$$
Z T=\frac{\sigma S^{2} T}{\kappa},
$$

where $\sigma$ is the electric conductance, $S$ is the Seebeck coefficient, $T$ is the absolute temperature, and $\kappa=\kappa_{e}+\kappa_{p}$ is the total thermal conductance that is usually split into electron and phonon contributions, respectively [1-3]. Generally speaking, materials with $Z T \approx 1$ are regarded as good thermoelectric components, while devices with a $Z T$ approaching or larger than 3 could efficiently compete with conventional energy conversion techniques. State of the art values for the figure of merit are about 1 , while higher values have been reported in the literature for particular materials which, however, have presently proven difficult to integrate into our technologies or to produce industrially in a reliable way, or whose cost makes them unaffordable at large scale [6]. Admittedly, the optimization of the figure of merit is a difficult problem. Indeed, an ideal thermoelectric material should hold the electric conductance and the Seebeck coefficient as high as possible, while keeping the thermal conductance as low as possible. Unfortunately, because of the Wiedemann-Franz law $\kappa_{e} / \sigma=\left(k_{B} \pi\right)^{2} T / 3 e^{2}$ (valid in a great extent for metals), where $k_{B}$ and $e$ are, respectively, the Boltzmann constant and carrier charge [7], the two conductances are locked together and increasing the first leads to an increase in the second. It therefore looked natural to attempt to decrease the phonon thermal conductance since this will hopefully not (greatly)

*roberto.dagosta@ehu.es affect the electronic properties, although the maximum $Z T$ achieved so far makes these devices not commercially viable.

After the seminal work by Hicks and Dresselhaus [8], strong research activity has been focused on nanostructured materials for thermoelectric applications. This boost can easily be explained as an attempt to escape from the Wiedemann-Franz law while dramatically increasing the electronic density of states $[9,10]$. With the discovery of graphene [11], and the subsequent investigation of its properties, it became apparent that graphene is not an efficient thermoelectric material since its thermal conductance is extremely high [12-14]. On the other hand, it has been shown that nanostructuring graphene with boron nitride in a nanoribbon increased the overall figure of merit by a factor of 20 [15]. Notwithstanding its phenomenal properties, the integration of graphene with actual silicon-based technologies has proven a quite challenging task, whose solution would probably require the complete redesign of electronics devices. As our present technology is based on silicon ( $\mathrm{Si}$ ) and germanium (Ge) semiconductors; it thus appears natural to look at the thermoelectric properties of these materials, since the integration of a thermoelectric device based on them would be simpler than the integration of carbon-based devices. For example, in silicon nanowires, the thermal conductance can be reduced in a factor of 100 due to the quenching of phonon transport and they exhibit a high thermoelectric conversion ratio [16]. This suggests a prospective avenue to improve the thermoelectric performance through decreasing the characteristic size of materials, and various nanostructures such as nanotubes and nanomembranes can be proposed.

Silicene resembles graphene [17-21] in the atomic single layer arrangements, i.e., it forms a honeycomb lattice and shares with the carbon system similar electronic properties. In particular, it is viewed as a new type of atomic-layered material with outstanding properties such as the zero effective mass at the Dirac point and infrared absorbance optical spectra [2224]. Experimentally, single layer silicene (buckled) [25-36] and silicene nanoribbons (SiNRs) [25,27] have been synthesized on Ag substrate. In particular, SiNRs up to a narrow width of $1.6 \mathrm{~nm}$ have been produced, aligned parallel to each other in a well-distributed way [25]. From the experience gained with the current microelectronics, we know that Ge 
is a good partner for $\mathrm{Si}$ since they share similar electronic properties and form bulk crystal with comparable lattice constant $\left(a_{\mathrm{Si}}=0.5431 \mathrm{~nm}\right.$, while $a_{\mathrm{Ge}}=0.5658 \mathrm{~nm}$, with a lattice mismatch of $4 \%$ ). The elastic limit is around $7 \%$. In the case of InAs, for instance, only one monolayer can be grown on GaAs [37]. A single layer hexagonal lattice of Ge, called germanene, has been predicted from ab initio calculations [22]. Theoretically, germanene presents a Dirac point, and the electronic and structural properties of this material would be very similar to those of silicene. We will discuss some of them in more detail in the following. In particular, we will consider germanene nanoribbons (GeNRs) of different widths and the possibility of forming Si-Ge single atomic layered nanoribbons by alternating stripes of $\mathrm{Si}$ with stripes of Ge.

In this paper, we investigate with an $a b$ initio technique combined with a linear response approach the thermoelectric properties of both two-dimensional (2D) silicene and germanene nanosheets and one-dimensional (1D) nanoribbons. We find that some of these systems will show a figure of merit larger than 1 at room temperature (with a maximum value of 2.18). Our results are consistent with those obtained by Pan et al. [38], although they are based on different techniques, especially for the calculations of the phonon thermal conductance. We believe that this agreement is partially fortuitous, as we will discuss in the following.

The paper is organized as follows. In Sec. II we will discuss in detail the numerical and theoretical methods we used to investigate the $\mathrm{Si}$ and $\mathrm{Ge}$ systems. In Sec. III, we introduce the 2D systems, silicene and germanene, study their stability, and investigate their transport properties. In Sec. IV, we investigate the $1 \mathrm{D}$ nanoribbons. In this section we focus mostly on the Ge system, since the SiNRs have been investigated elsewhere, and from our calculations, Si and Ge nanoribbons do share essentially some of the same properties. We find that the nanoribbons can have a quite large figure of merit. This is due to the fact that both $\mathrm{Si}$ and $\mathrm{Ge}$ nanoribbons have a finite electronic gap that dramatically enhances the Seebeck coefficient. In Sec. V, we consider nanoribbons created by alternating stripes of $\mathrm{Si}$ and $\mathrm{Ge}$. By nanostructuring the nanoribbons we would like to confine the phonons and therefore decrease the thermal conductance. However, we report that the SiGeNRs do show some the same transport properties of the pure $\mathrm{Si}$ or pure Ge nanoribbons. This is due to the limitations of our method of choice, namely, a full $a b$ initio study for the phonon energy transport. Indeed, within this technique we are limited to fairly small nanoribbons and therefore the long-wavelength phonons are not quenched by the regular pattern of the structured nanoribbons. On the other hand, a classical technique, based on molecular dynamics, would allow us to calculate the thermal conductance of larger devices. However, this technique does not recover the correct quantum limit of these one-dimensional systems, and therefore we do expect that the molecular dynamics results to give the incorrect thermal conductance at a temperature below the Debye temperature, which for $\mathrm{Si}$ and Ge systems can be estimated to be about 640 and $374 \mathrm{~K}$, respectively. We check the idea that nanostructuring would decrease the phonon thermal conductance by using a tight-binding approximation, which allows us to consider a larger supercell than a purely $a b$ initio method. We indeed show that the thermal conductance greatly decreases when we consider a heterostructure of $\mathrm{Si}$ and Ge. Finally, in Sec. VI we draw our conclusions and give some outlooks of this work.

\section{METHOD}

In linear response theory, by using Onsager's relations and the Landauer theory of quantum transport, the electrical conductance $\sigma$, the Seebeck coefficient $S$, and the electron contributed thermal conductance $\kappa_{e}$, can be written as $[2,3,39]$

$$
\begin{gathered}
\sigma_{\alpha \beta}(\mu, T)=e^{2} L_{00}(\mu, T), \\
S_{\alpha \beta}(\mu, T)=\frac{1}{e T} \frac{L_{01}(\mu, T)}{L_{00}(\mu, T)}, \\
\kappa_{e, \alpha \beta}(\mu, T)=\frac{1}{T}\left[L_{11}(\mu, T)+\frac{L_{01}(\mu, T)^{2}}{L_{00}(\mu, T)}\right],
\end{gathered}
$$

where

$$
L_{m n}(\mu, T)=-\frac{1}{A} \int_{-\infty}^{\infty} d \epsilon \mathcal{T}_{e, \alpha \beta}(\epsilon)(\epsilon-\mu)^{m+n} \frac{\partial f(\epsilon, \mu, T)}{\partial \epsilon}
$$

is the Lorenz integral. In these equations, $\mu$ is the chemical potential, $A$ is the area of the considered system, $\alpha$ and $\beta$ are the indices of the spatial components $x, y$, and $z, f(\epsilon, \mu, T)$ is the Fermi distribution function at a given temperature $T$, and $\mathcal{T}_{e}$ is a transmission function which is related to the probability of electrons to cross the system [40,41]. Similarly, the phonon thermal conductance is given by [42]

$$
\kappa_{p, \alpha \beta}(T)=\frac{1}{A} \int_{0}^{\infty} d \omega \mathcal{T}_{p, \alpha \beta}(\omega) \hbar \omega \frac{\partial n(\omega, T)}{\partial T},
$$

where $\omega$ is the phonon-vibrational frequency, $\hbar$ is the reduced Planck constant, and $n(\omega, T)$ is the Bose-Einstein distribution function. Again, $\mathcal{T}_{p}$ is a transmission function for phonons. A common expression of the electron and phonon transmission functions can be given in terms of the electron and phonon band structures, respectively,

$$
\mathcal{T}_{e / p, \alpha \beta}(E)=\frac{1}{N} \sum_{i, \mathbf{k}} \tau_{e / p, i, \mathbf{k}} v_{\alpha}(i, \mathbf{k}) v_{\beta}(i, \mathbf{k}) \mathcal{D}_{e / p}\left(E_{i, \mathbf{k}}\right),
$$

where $N$ is the number of sampled $\mathbf{k}$ points in the first Brillouin zone, $i$ is the band index, $\tau_{e / p}$ is the relaxation time of electrons/phonons, $v$ is the velocity calculated from the band dispersion, and $\mathcal{D}_{e / p}\left(E_{i, \mathbf{k}}\right)$ is the electron/phonon density of states associated with band $i$.

To obtain the energy band structure, we perform firstprinciples calculations within the local density approximation by using the projector-augmented wave potentials as implemented in VASP [43]. The exchange-correlation energy is chosen in the form of Ceperley-Alder which has been parametrized by Perdew and Zunger [44,45]. For the selfconsistent potential and the total energy calculations, the $\mathbf{k}$ points of the Brillouin zone in the reciprocal space are sampled by a $(25 \times 1 \times 1)$ Monkhorst-Pack grid. The kinetic energy cutoff is set to $500 \mathrm{eV}$. After ionic relaxation, the HellmannFeynman forces acting on each atom are less than $0.01 \mathrm{eV} / \AA$. We obtain the force-constant matrix for the calculation of the 
phonon dispersion, through the small displacement method [46]. We use a supercell technique with $15 \AA$ of vacuum. In these calculations we have neglected both the phonon-phonon and the electron-phonon interactions. We expect that for the low-energy phonons, mostly responsible for the thermal transport, the correction due to these interactions will be small, especially for the Seebeck coefficient.

In the following, we will consider both the figure of merit of Eq. (1) and the electronic figure of merit $Z T_{e}$, defined as

$$
Z T_{e}=\frac{S^{2} \sigma}{\kappa_{e}} T
$$

Then Eq. (1) can be rewritten as

$$
Z T=\frac{S^{2} \sigma}{\kappa_{p}+\kappa_{e}} T=\frac{S^{2} \sigma}{\kappa_{e}} T\left(\frac{1}{1+\kappa_{p} / \kappa_{e}}\right)=\frac{Z T_{e}}{1+\kappa_{p} / \kappa_{e}} .
$$

Although $Z T_{e}$ is not a physical measurable quantity, it is useful because it provides an upper bound to the total figure of merit, and since it does not include the phonon thermal contribution is easier to calculate. A small $Z T_{e}$ will therefore imply a small figure of merit $Z T$.

\section{SILICON AND GERMANIUM MONOLAYERS}

We first investigate the electronic properties of a single layer of silicon, i.e., silicene. In trying to closely reproduce the experimental setup [29,32,33], we put one layer of $3 \times 3$ silicene on top of five layers of $4 \times 4 \mathrm{Ag}(111)$ : According to experimental evidence, the two lattices should match, thus decreasing the total stress at the boundary and creating an ideal supercell for our calculations. The geometrical structure for silicene obtained after the full relaxation is shown in Fig. 1(a) and corresponds to the structure discussed in Ref. [36]. We have superimposed the $\operatorname{Ag}(111)$ layer to show the excellent structural matching, as highlighted by the boundary continuous (red) line. Figure 1(b) shows the silicene obtained by removing the silver substrate in Fig. 1(a). Contrary to graphene, silicene is not a strict two-dimensional system, in the sense that the atoms in silicene are arranged on two atomic layers with a fairly small buckling distance, which depends on the presence of the substrate. Indeed, it is found that the atomic arrangement is further distorted by the metallic substrate [36]. Starting from a single layer of silicon, arranged in a plane on a hexagonal lattice without the Ag substrate, we would have obtained a system with a different buckling, where the atoms would divide equally between the upper and lower planes. In our optimized structure however, we observe that the silicene presents buckling forming two atomic layers with six atoms on top of the other 12 atoms which are therefore closer to the Ag surface. The buckling distance between these two layers is about $0.79 \AA$. In Fig. 1(c) the electronic energy band for the distorted silicene is plotted along the high-symmetry points of the first Brillouin zone, where the dotted line indicates the Fermi energy that we set for convenience at 0 . It can be seen that a band gap of about $0.3 \mathrm{eV}$ crosses the Fermi energy, indicating semiconducting properties of the system. This must be compared with the flat silicene (unoptimized structure) and the silicene optimized without the Ag substrate, which both (a)

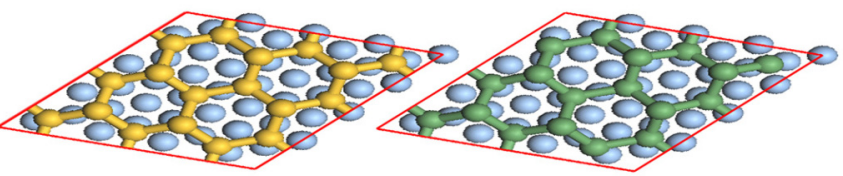

(b)

(c)

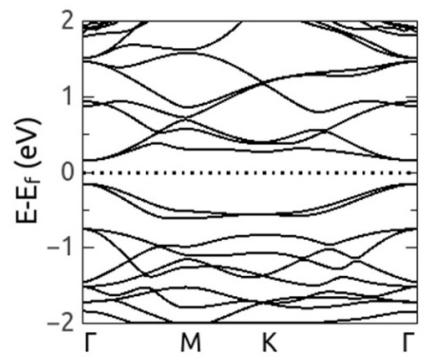

(e)

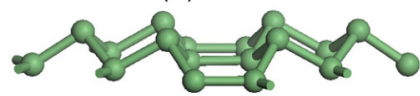

(f)

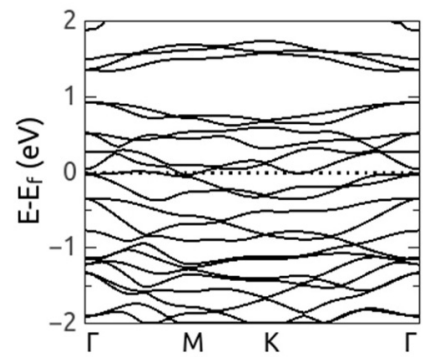

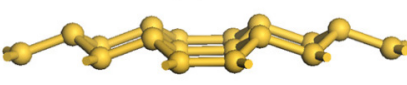

FIG. 1. (Color online) Geometrical structures of one-layer $3 \times 3$ (a) silicene and (d) germanene on top of five-layer $4 \times 4 \mathrm{Ag}(111)$. (b), (e) Distorted silicene and germanene obtained by removing the silver substrate from (a) and (d), respectively. (c), (f) Electronic energy bands corresponding to the distorted silicene and germanene grown on $\operatorname{Ag}(111)$, respectively, where the dotted line denotes the Fermi energy.

present a Dirac point at the $K$ point of the first Brillouin zone, therefore both showing metallic properties (see Fig. 3). A detailed discussion of the electronic structure of supported silicene can be found in Ref. [36].

Germanene is an analog of silicene, where the silicon atoms are replaced by germanium. Although, to the best of our knowledge, up to now there is no direct experimental observation of these structures, here we study the electronic properties of two-dimensional germanene. Figure 1(d) shows the atomic structure of one-layer $3 \times 3$ germanene on top of five-layer $4 \times 4 \mathrm{Ag}(111)$, and Fig. 1(e) shows the unsupported single-layer germanene by removing the silver substrate. The structure has been fully relaxed. It is found from Fig. 1(e) that similar to silicene, two layers are formed with six Ge atoms on the top layer and the other $12 \mathrm{Ge}$ atoms on the bottom layer closer to the Ag surface. The buckling distance between the two layers is about $1.42 \AA$. Figure 1 (f) shows the band structure of the distorted germanene without the Ag substrate. It is found that there is no gap through the Fermi energy, indicating metallic properties. The zero gap observed in germanene originates from the high-buckling distance between the two atomic layers.

Based on the energy bands, we have calculated the thermoelectric coefficients of the two-dimensional silicene and germanene structures at room temperature, $T=300 \mathrm{~K}$. We have used the BOLTZTRAP code [47] to perform the integration over the points in momentum space in the first Brillouin zone obtained from the VASP calculations. To calculate the electronic figure of merit we have evaluated the transport coefficients given in Eqs. (2)-(4) in the constant relaxation time approximation and by assuming $1 / \tau_{e}$ to be proportional 

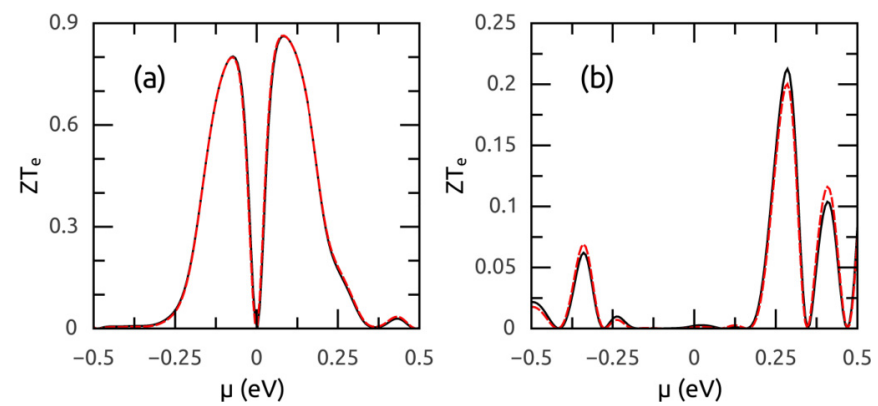

FIG. 2. (Color online) (a), (b) Dimensionless electronic figure of merit $Z T_{e}$ at room temperature as a function of chemical potential $\mu$ corresponding to the unsupported distorted silicene and germanene, respectively, evaluated in the constant relaxation time approximation (black continuous line) and for $1 / \tau_{e} \propto E$ (red dashed line).

to the energy $[20,48]$. We found that both approximations give essentially the same results, as we show in Figs. 2 and 4. There the continuous black line represents the result in the constant relaxation time, and the dashed red line the result in the inverse energy dependence. The observation that the figure of merit is independent from the relaxation time stems from the form of the Lorentz integral, where the derivative of the Fermi function is a strongly localized function around the Fermi energy. Therefore, the relaxation time is always evaluated only around the Fermi energy and can be replaced with its value at that point. As we discussed in the Introduction, we provide an upper bound to $Z T$, in the form of the electronic figure of merit $Z T_{e}$. Figures 2(a) and 2(b) show the dimensionless electronic figure of merit $Z T_{e}$ as a function of the chemical potential $\mu$ for the distorted silicene and germanene, respectively. It can be seen that the figure of merit for silicene exhibits two peaks in the left- and right-hand sides of $\mu=0$, which separately correspond to hole and electron transport. The maximum of the peak is $Z T_{e} \simeq 0.81$, while for the unsupported germanene, it can be seen from Fig. 2(b) that the peak of $Z T_{e}$ is very small at $\mu=0$, although some peaks appear at $\sim \pm 0.3 \mathrm{eV}$. The reason is that the unsupported germanene has a metallic character which leads to a very small Seebeck coefficient.

In Fig. 3 we show the electronic properties of freestanding silicene and germanene. After the full relaxation, it is found that the buckling distance for silicene is about $0.43 \AA$, and for germanene $0.65 \AA$. For both the freestanding silicene and germanene, from Figs. 3(a) and 3(b) it can be seen that there is (a)

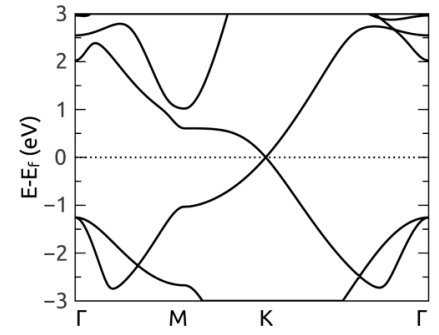

(b)

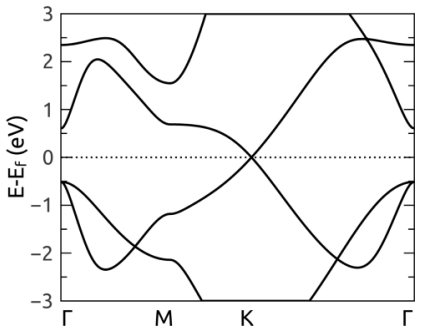

FIG. 3. Electron energy bands of freestanding (a) silicene and (b) germanene, respectively, where the dotted line denotes the Fermi energy.
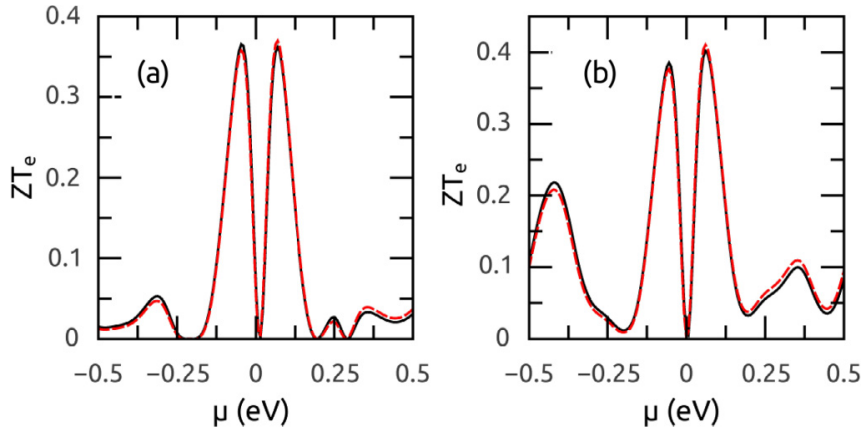

FIG. 4. (Color online) (a), (b) Dimensionless electronic figure of merit $Z T_{e}$ at room temperature as a function of chemical potential $\mu$ for the freestanding silicene and germanene, respectively, evaluated in the constant relaxation time approximation (black continuous line) and for $1 / \tau_{e} \propto E$ (red dashed line).

no gap at the Fermi energy. Indeed, at the high-symmetry point $K$, a linear energy dispersion is shown in the band structures, indicating the existence of the massless Dirac fermions in these low-dimensional Si structures [22] similar to the graphene [17].

Through the energy band structure calculations, in Fig. 4 we investigate the dimensionless electronic figure of merit $Z T_{e}$ for both freestanding silicene and germanene. It is found that the figure of merit for silicene and germanene shows two peaks near $\mu=0$. The maximum value of $Z T_{e}$ is 0.36 [see Fig. 4(a)], while the maximum of the peak for germanene is 0.41 [see Fig. 4(b)].

We have shown that silicene and germanene, crystal structures similar to graphene where carbon is replaced by either silicon or germanium, might possibly have a figure of merit of the order of 1 . Our calculations provide an upper limit to the theoretical figure of merit since in these calculations we are not including the phonon thermal conductance and suggest that silicene might have better thermoelectric properties in this 2D system since it presents a gap in the electronic energy spectrum which corresponds to a large Seebeck coefficient.

\section{QUASI-ONE-DIMENSIONAL NANOSTRUCTURES}

We now consider quasi-one-dimensional systems, i.e., nanoribbons, made of stripes of germanene or silicene of finite width. We assume that it is possible to "cut" those stripes from the respective crystal by removing the excess material. It has been reported that SiNRs can have a quite large figure of merit, up to 5 at $600 \mathrm{~K}$ [38]. Motivated by these results, and by the expectation that germanene nanoribbons might perform better since their Debye temperature is lower, we have investigated the thermoelectric efficiency of GeNRs and, in the next session, nanoribbons obtained by alternating $\mathrm{Si}$ and $\mathrm{Ge}$ nanostructures or by randomizing the $\mathrm{Si}$ and $\mathrm{Ge}$ arrangements. As standard with nanoribbons, there are two ways to terminate the edges of the ribbons (see Fig. 5), forming either zigzag or armchair edges. We identify the quantities associated with the zigzag with a $Z$ and those of the armchair with an $A$. As to these one-dimensional systems, the electrical conductance $\sigma$, Seebeck coefficient $S$, and thermal conductance could be contracted into a scalar instead of a 

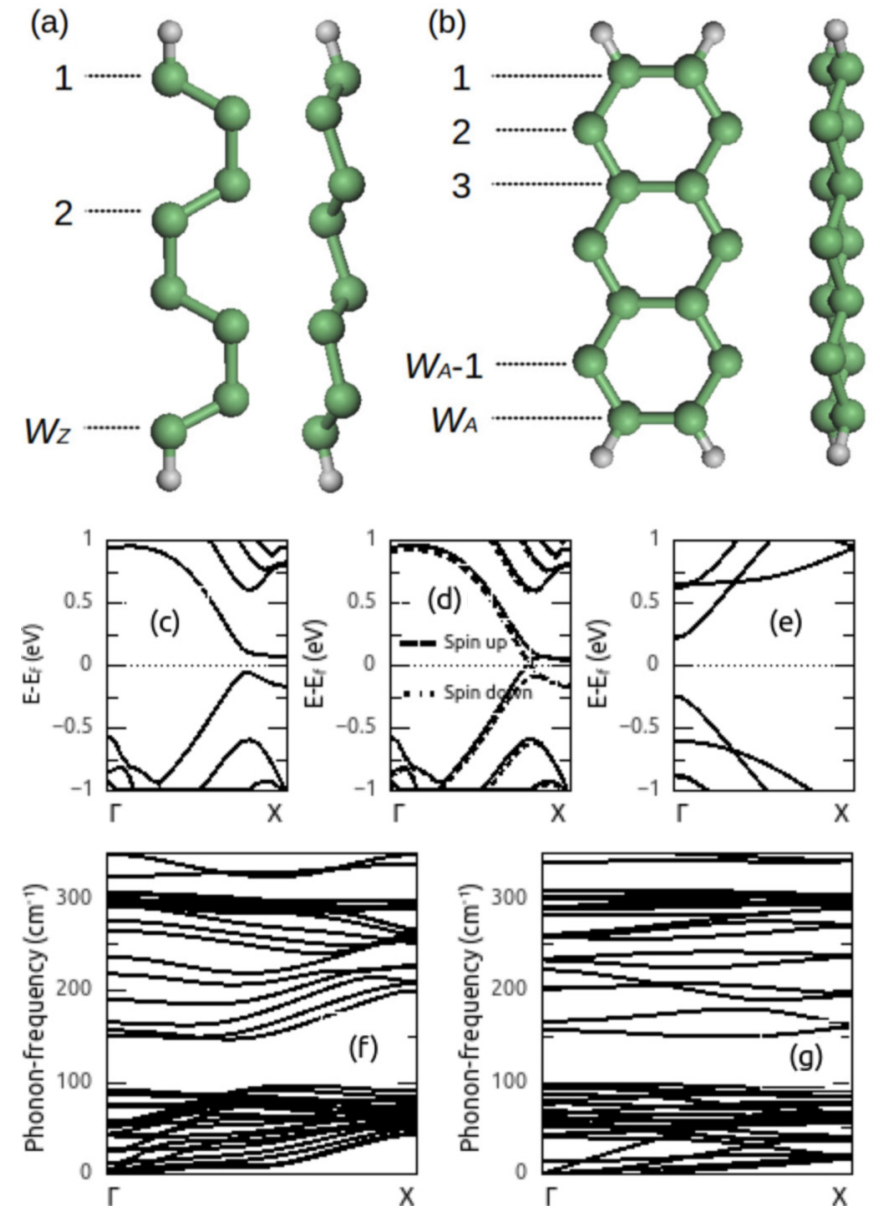

FIG. 5. (Color online) (a), (b) Optimized geometrical structures of $Z$-GeNRs and $A$-GeNRs and their lateral views. For the atoms at the edges, we passivate the unsaturated bonds with hydrogen atoms. $W_{Z}$ and $W_{A}$ denote the width of the nanoribbons for the zigzag- and armchair-terminated nanoribbons, respectively. (c) Electron energy band for $Z$-GeNRs with $W_{Z}=6$ for the AFM state. Notice the presence of a small electronic gap. (d) Electron energy band for $Z$-GeNRs with $W_{Z}=6$ for the FM state. (e) Electron energy band of $A$-GeNRs with $W_{A}=6$ corresponding to the NM state. In (c)-(e) the Fermi energy is chosen as the reference energy and set to 0 . (f), (g) Phonon energy dispersions for $Z$-GeNRs with $W_{Z}=6$ and $A$-GeNRs with $W_{A}=6$, respectively.

tensor, and the $\alpha$ and $\beta$ are fixed in the $x$ direction (see details in Ref. [15]). Therefore in this case we can calculate the electron and phonon transmissions $\mathcal{T}_{e / p}$ by counting the number of transport modes from the energy bands. This gives the same results by comparing them with the constant relaxation time approximation in Eq. (7) except by a factor difference, that is immaterial for the evaluation of the figure of merit.

\section{A. Germanene nanoribbons}

Figures 5(a) and 5(b) show the optimized structures of zigzag- and armchair-edged GeNRs (Z-GeNRs and $A$ GeNRs), respectively. To see the buckling more clearly, we report here a side view of these structures. Hydrogen atoms are used to passivate the unsaturated bonds of the Ge atoms at the edges. $W_{Z}$ and $W_{A}$ identify the ribbon width. It can be seen from the top view that GeNRs form hexagonal rings as the union of two sublattices, but, at odds with what happens for graphene nanoribbons, atoms in these two sublattices do not belong to the same plane: In the vertical direction there is some buckling, which is almost uniform for the atoms at the edge or in the center. Our calculations give for the Z-GeNRs a buckling distance of $0.62 \AA$, while for $A$-GeNRs they give $0.66 \AA$. For these nanoribbons, our total energy calculations show that the antiferromagnetic (AFM) state of $Z$-GeNRs is more stable than the ferromagnetic (FM) and nonmagnetic (NM) state counterparts. This is in agreement with other calculations performed for SiNRs [38] and theoretical predictions originally derived for graphene, which we expect to be valid for these systems [49]. However, the energy difference between the different magnetic phases is small. This might be important for device stability, especially at temperatures higher than $300 \mathrm{~K}$. The bands of AFM and FM states are shown in Figs. 5(c) and 5(d), respectively, where the dotted line corresponds to the Fermi energy. We can see that the AFM state exhibits a finite small gap: The bands for spin up and down are degenerate and the gap is about $0.1 \mathrm{eV}$, while for the FM state, it is found that spin up and down are nondegenerate, producing metallic properties, and similar properties are valid for the NM state (not shown). In the case of $A-\mathrm{GeNR}$, our calculations indicate that the NM state is stable, indicating semiconducting properties as shown in Fig. 5(e). Because the metallic system produces bad thermoelectric properties (generally the $Z T$ is smaller than 0.1 ), in the rest of this work, we will focus our attention on the AFM state in the zigzag-edged nanoribbons and the NM state in the armchair-edged nanoribbons. To confirm the structural stability of GeNRs, we have calculated the phonon dispersion relations. In Figs. 5(f) and 5(g) we report the phonon dispersion relation for the nanoribbons with width 6 for both $Z$-GeNR and $A$-GeNR, i.e., $W_{Z}=W_{A}=6$, respectively. It can be seen that in the limit of $\omega \rightarrow 0$, there are four acoustic phonon modes in the spectrum stemming from the lattice symmetry. In particular, no negative phonon mode is observed, which confirms that both the $Z$-GeNRs and $A$-GeNRs passivated by hydrogen are structurally stable.

To calculate the figure of merit $Z T$, we begin with the electron transport properties. Figures 6(a) and 6(b) show the transmission coefficient $\mathcal{T}_{e}$ as a function of the electron energy $E$ for both $Z$-GeNRs and $A$-GeNRs, respectively. It can be seen that $\mathcal{T}_{e}$ exhibits a clear quantum stepwise structure, due to opening and closing of elastic transmission channels: Notice that the jumps are quantized and equal to 2 due to the electron spin. More interesting, a monotonously decreasing band gap is observed in the Z-GeNRs with the increasing of the ribbon width [see Fig. 6(c)]. This must be compared with the oscillatory behavior we observe for the $A$-GeNRs [see Fig. 6(d)]. For the $A-G e N R s$, for the ribbon widths $W_{A}=3 p$ and $3 p+1$ (where $p$ is positive integer), the gap is larger than that of the ribbon width $W_{A}=3 p+2$. By making use of the transmission probability, using Eqs. (2) and (3), we can calculate the electrical conductance $\sigma$, Seebeck coefficient $S$, and electron contributed thermal conductance $\kappa_{e}$. In Figs. 6(e) and 6(f), the electrical conductance as a function of chemical potential is plotted for both Z-GeNRs and $A$-GeNRs, respectively. It can be seen that the electrical 

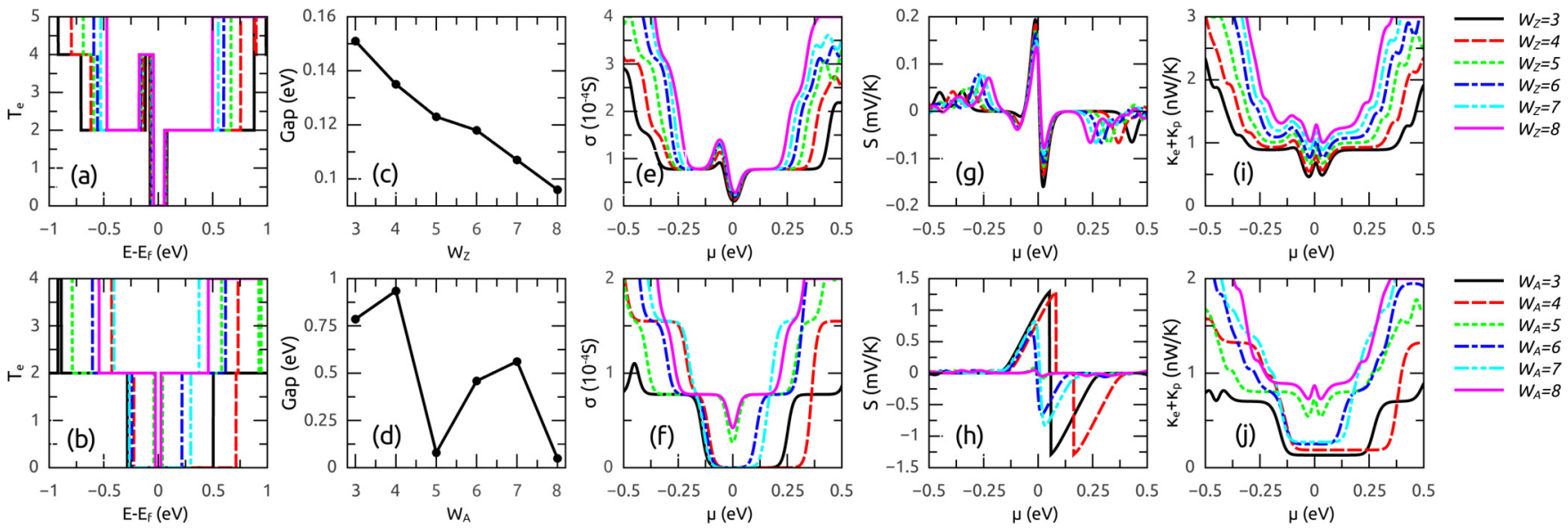

FIG. 6. (Color online) (a), (b) Electron transmission coefficient as a function of energy for $Z$-GeNRs and $A$-GeNRs with various ribbon width, respectively. (c), (d) Band gap of $Z$-GeNRs and $A$-GeNRs as a function of the ribbon widths $W_{Z}$ and $W_{A}$, respectively. (e), (f) Electrical conductance. (g), (h) Seebeck coefficient. (i), (j) Electron and phonon thermal conductances for $Z$-GeNRs and $A$-GeNRs versus chemical potential $\mu$, where the temperature is set at $300 \mathrm{~K}$.

conductance for zigzag nanoribbons gradually increases with the ribbon width, and there is a peak corresponding to the transmission step at $E \approx 0.5 \mathrm{eV}$. Around the Fermi energy, the conductance vanishes due to the finite gap. For the $A-\mathrm{GeNRs}$, we find that the electrical conductance for the ribbon with width $3 p$ or $3 p+1$ vanishes, while for the ribbon with width $3 p+2$, a nonzero dip is found. Interestingly, the conductance for all the curves of $A$-GeNRs exhibits quantized plateaulike characteristics.

In Figs. 6(g) and 6(h) we report the Seebeck coefficient as a function of the chemical potential $\mu$. It can be seen from Fig. $6(\mathrm{~g})$ that $S$ presents two peaks around the position of the chemical potential needed to overcome the gap. Moreover the two peaks show different signs with positive and negative values. This behavior indicates the different carrier transport: The positive sign in the region of $\mu<0$ corresponds to hole transport, while the negative sign at $\mu>0$ corresponds to electron transport. In addition the absolute value of the peak of the Seebeck coefficient decreases with increasing $W_{Z}$. In the case of $A$-GeNRs, it is found [see Fig. 6(h)] that for the nanoribbons with widths $3 p$ and $3 p+1$, the two Seebeck coefficient peaks with opposite sign can also be found centered around zero value of the chemical potential. We note that for the nanoribbons with width $3 p+2$, the Seebeck coefficient is very small due to the small electronic gap. In Figs. 6(i) and $6(\mathrm{j})$ the total thermal conductance for $Z$-GeNRs and $A$-GeNRs is depicted, respectively. It can be seen that the thermal conductance for $Z$-GeNRs increases with increasing the width of the nanoribbon. By checking the variation of the electrical and thermal conductances $\sigma$ and $\kappa$, it is found that corresponding to the dip position of the electrical conductance, the electric thermal conductance (and therefore the total thermal conductance) shows a peak which becomes sharper with increasing $W_{A}$. Moreover, a similar effect can also be found in the $A$-GeNRs with width $3 p+2$ as shown in Fig. 6(j).

To study the lattice thermal transport properties, the supercell approach is utilized to calculate the phonon force constant and then the dispersion relation is obtained by diagonalizing the corresponding dynamical matrix [46]. In
Figs. 7(a) and 7(b), the phonon thermal conductance $\kappa_{p}$ as a function of temperature $T$ for both $Z$-GeNRs and $A$-GeNRs is plotted, respectively. It can be seen that the phonon thermal conductance increases with increasing the temperature, and finally reaches a constant value corresponding to the classical limit when $T>400 \mathrm{~K}$. Moreover the thermal conductance for wide nanoribbons exhibits a higher value than that of the narrow nanoribbons. This can simply be explained by counting the number of phonon channels, because the wide nanoribbons should have more phonon channels contributing to the thermal transport. To show the behavior at low temperatures of the phonon thermal conductance, in Fig. 7(c), we plot the logarithm of $\kappa_{p}$ versus the logarithm of $T$. It can be seen that $\kappa_{p}$ shows a linear dependence on the temperature at low $T, T<20 \mathrm{~K}$. At low temperature, for the one-dimensional systems, the lattice thermal conductance is dominated by the low-frequency acoustic phonons, and Eq. (4) can be recast as

$$
\kappa_{p}(T)=\frac{4 k_{B}^{2} T}{h} \int_{0}^{\infty} d \xi \xi^{2} \frac{e^{\xi}}{\left(e^{\xi}-1\right)^{2}}=\frac{2 \pi k_{B}^{2} T}{3 \hbar},
$$

where $\xi=\frac{\hbar \omega}{k_{B} T}$ and we have approximated the transmission probability $\mathcal{T}_{p}(\omega)=4$ because of the sum rule. According to this approximation, it can be seen that the phonon thermal conductance exhibits a linear dependence on $T$ in quasi-onedimensional systems [42].

By combining the results of the electron and phonon calculations, we can finally investigate the thermoelectric efficiency of the GeNRs. Figures 8(a) and 8(b) report the thermoelectric figure of merit $Z T$ as a function of the ribbon width for both $Z$-GeNRs and $A$-GeNRs, respectively. Here $Z T$ is the maximum value of the figure of merit with respect to the chemical potential near the Fermi energy. It can be seen from Fig. 8(a) that at narrow Z-GeNRs, $Z T$ for electron and hole is about 0.35 and 0.61 , and then it decreases with increasing the ribbon width. This effect can be explained by the lessening of the Seebeck coefficient and growing of the thermal conductance outweighing the increasing electrical conductance. Moreover, we observe from Fig. 8(b) for the 

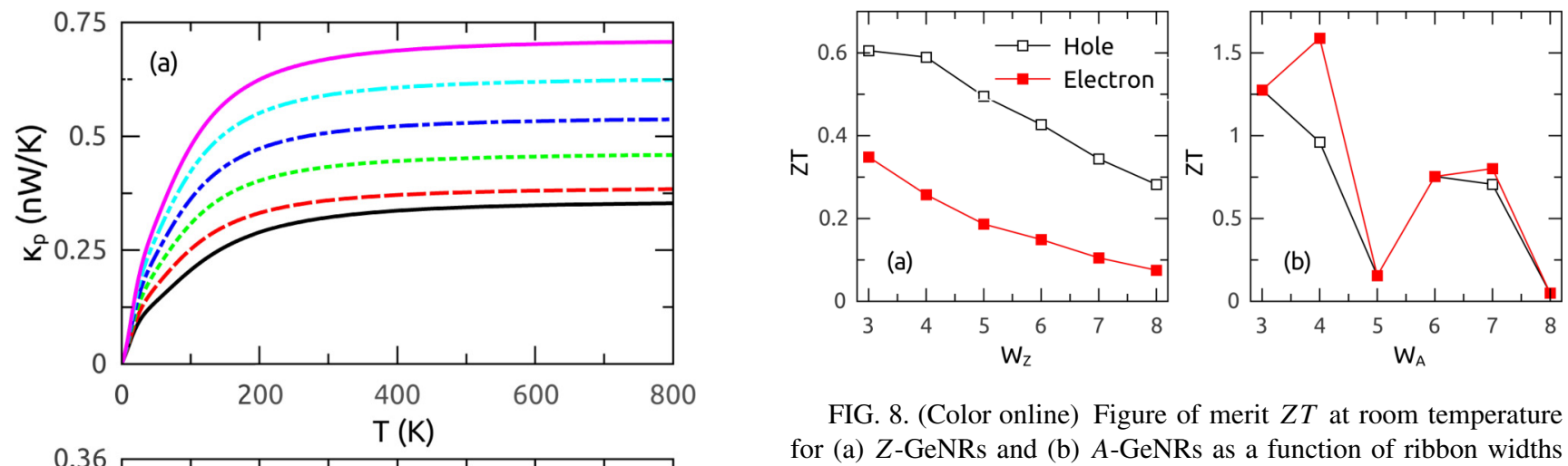

FIG. 8. (Color online) Figure of merit $Z T$ at room temperature for (a) $Z$-GeNRs and (b) $A$-GeNRs as a function of ribbon widths $W_{Z}$ and $W_{A}$, respectively. In black (square hollow points) we report the peak value of $Z T$ at negative values of the chemical potential $\mu$ associated with the hole transport, and in red (square full points) the peak value of $Z T$ associated with the electron transport (positive $\mu$ ).

To calculate the hole carrier density we used

$$
n_{p}=\int_{-\infty}^{E_{v}} d \epsilon[1-f(\epsilon, \mu, T)] \mathcal{D}(\epsilon),
$$

where $E_{v}$ is the top of the valence band. We report our results for the electron and hole carrier densities for the zigzag GeNRs in Table I, where $\mu_{M}$ is the chemical potential of the maximum figure of merit.

Similarly, we report our results for the electron and hole carrier densities in Table II for the armchair GeNRs.

Our results are consistent with what has been found for SiNRs [38]. However, we would like to point out that from our calculations the phonon thermal conductance of the small GeNR is never negligible with respect to the electron thermal conductance, as instead has been argued for the SiNRs in Ref. [38]. We believe this is an artifact of the classical methods used in Ref. [38]. Unlike our quantum simulations, in these quasi-one-dimensional systems that are in the ballistic thermal transport regime, classical methods would not recover the linear dependence of phonon thermal conductance at low temperatures. Moreover, the classical calculations should be valid only for temperatures higher than the Debye temperature, which for these systems can be estimated to be about $600 \mathrm{~K}$ for the silicene nanoribbons. In Ref. [38] the classical calculations are instead used to evaluate the phonon thermal conductance also below the Debye temperature, an assumption that would need an explanation. At the same time, the quantum technique does not include any inelastic effect and it is greatly limited

coefficients show an oscillatory behavior. In the case of the nanoribbons with widths $W_{A}=3 p$ and $3 p+1, Z T$ is larger than 1 for narrow nanoribbons. In particular, for the ribbon width $W_{A}=4$, the $Z T$ reaches up to 1.63 , indicating a high thermoelectric conversion efficiency in these nanostructures. We have calculated the carrier density at the chemical potential that gives the maximum efficiency for the SiNRs and the GeNRs. To do that we used the formulas valid for the electron carrier density in an intrinsic semiconductor

$$
n_{e}=\int_{E_{c}}^{\infty} d \epsilon f(\epsilon, \mu, T) \mathcal{D}(\epsilon),
$$

where $f(\epsilon, \mu, T)$ is the Fermi distribution, $\mathcal{D}(\epsilon)$ is the density of states, and $E_{c}$ is the bottom energy of the conduction band.

TABLE I. Electron carrier density for the zigzag nanoribbons as a function of the width calculated at the chemical potential that gives the maximum figure of merit, at $T=300 \mathrm{~K}$. The $e$ and $h$ subscripts refer to the electrons and holes transport, respectively.

\begin{tabular}{lcccccc}
\hline \hline$W_{z}$ & 3 & 4 & 5 & 6 & 7 & 8 \\
\hline$\mu_{M, e}(\mathrm{meV})$ & 32.5 & 32 & 32 & 31.5 & 31 & 31.5 \\
$n_{e}\left(10^{12} \mathrm{~cm}^{-2}\right)$ & 6.74 & 6.85 & 7.05 & 6.57 & 6.18 & 6.24 \\
$\mu_{M, p}(\mathrm{meV})$ & -24 & -22 & -20 & -19.5 & -18 & -16 \\
$n_{p}\left(10^{12} \mathrm{~cm}^{-2}\right)$ & 4.15 & 3.65 & 3.13 & 3.42 & 2.65 & 2.71 \\
\hline \hline
\end{tabular}


TABLE II. Hole carrier density for the armchair nanoribbons as a function of the width calculated at the chemical potential that gives the maximum figure of merit, at $T=300 \mathrm{~K}$. The $e$ and $h$ subscripts refer to the electron or hole transport, respectively.

\begin{tabular}{lcccccc}
\hline \hline$W_{A}$ & 3 & 4 & 5 & 6 & 7 & 8 \\
\hline$\mu_{M, e}(\mathrm{meV})$ & 231 & 337 & 22.5 & 90 & 131 & 20 \\
$n_{e}\left(10^{12} \mathrm{~cm}^{-2}\right)$ & 0.60 & 3.53 & 2.24 & 0.797 & 0.75 & 1.97 \\
$\mu_{M, p}(\mathrm{meV})$ & -120 & -91 & -22 & -102 & -113 & -20 \\
$n_{p}\left(10^{12} \mathrm{~cm}^{-2}\right)$ & 1.12 & 2.26 & 2.37 & 0.937 & 0.875 & 1.88 \\
\hline \hline
\end{tabular}

in size, i.e., we cannot consider a large supercell as instead is possible with classical methods [38].

\section{B. Silicene nanoribbons}

For completeness, and to have a direct comparison with the results available in the literature [38], we have calculated the figure of merit of SiNRs similar to the GeNRs we have investigated in the previous section. Here we report only the phonon thermal conductance and the figure of merit. The electron transport coefficients $\sigma, S$, and $\kappa_{e}$ have shapes similar to those in Fig. 6 and we do not show them again. We plot in Figs. 9(a) and 9(b), the phonon thermal conductance $\kappa_{p}$ for both zigzag- and armchair-edged SiNRs (Z-SiNRs and $A$-SiNRs) as a function of temperature $T$, respectively. These plots should be compared to the results of Table 1 of Ref. [38]. We could see that we obtain a larger thermal conductance at $300 \mathrm{~K}$. As to the armchair nanoribbons, the thermal conductance is also increased except for the ribbon widths $W_{A}=3$ and 4 whose values are indeed close [see Fig. 9(b)].

In Fig. 10 the figure of merit for SiNRs as a function of ribbon width is shown. It can be seen that the figure of merit for $Z$-SiNRs decreases with the increase of the ribbon width. Moreover the $Z T$ for the hole transport is larger than that contributed from the electron transport. The reason is due to the increased phonon thermal conductance and decreased electronic band gap. For the armchair nanoribbons, it is found from Fig. 10(b) that the figure of merit at the narrow ribbon is quite large, about 1.04. With the increase of the ribbon width, the $Z T$ decreases in magnitude and exhibits an oscillatory behavior. We notice that, due to the larger thermal conductance
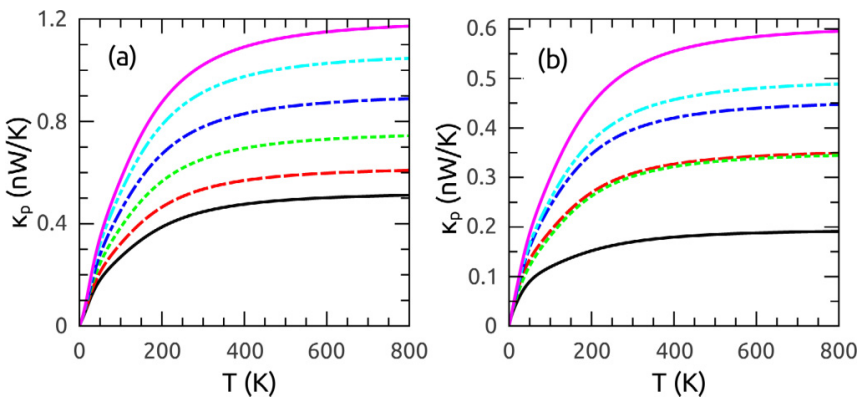

FIG. 9. (Color online) Phonon thermal conductance $\kappa_{p}$ of (a) $Z$ SiNRs and (b) $A$-SiNRs as a function of temperature, respectively, where each curve corresponds to a specified ribbon width as shown in Fig. 6.
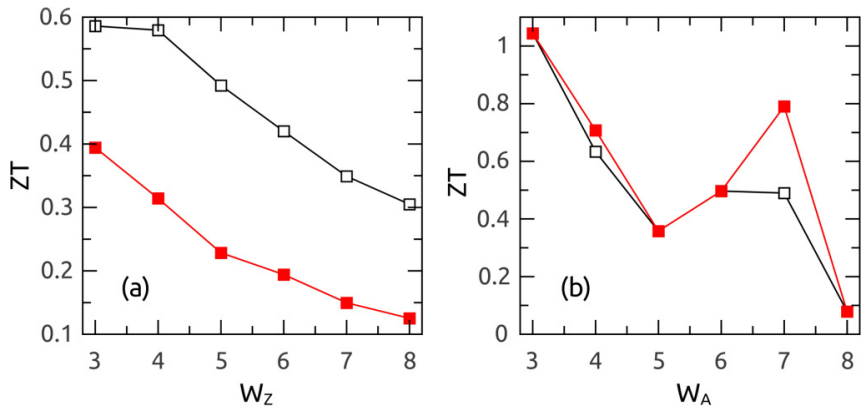

FIG. 10. (Color online) Figure of merit $Z T$ at room temperature for (a) $Z$-SiNRs and (b) $A$-SiNRs as a function of ribbon widths $W_{Z}$ and $W_{A}$, where the black square hollow points and the red square full points correspond to the hole and electron transport, respectively.

we obtain a figure of merit of the SiNRs that is smaller than the one reported in Ref. [38].

\section{SILICON-GERMANIUM HETEROSTRUCTURES}

We have shown that the $\mathrm{Si}$ and Ge nanoribbons can have a substantial figure of merit, which is slightly above 1 . On the other hand, we would like to explore the possibility of improving on this result by nanostructuring these nanoribbons. Since $\mathrm{Si}$ and $\mathrm{Ge}$ nanoribbons do share similar electronic properties, our first attempt is to investigate a nanoribbon created by alternating stripes of $\mathrm{Si}$ and $\mathrm{Ge}$ in the direction of the growth of the nanoribbon. Hopefully, their different masses would create a trap for the phonon modes thus reducing the thermal conductance of the device and improving the overall figure of merit $Z T$. We will show in the following section that this idea is working partially and we do have a modest increasing of $Z T$. This is a limitation of our quantum method of calculating the thermal conductance, since we are restricted in the size of the supercell we can consider for our calculations. Indeed, the low-energy phonons responsible primarily for the thermal transport have a wavelength that spans many supercells thus making the chemical modulation ineffective as a phonon trap. To improve on this result, we have therefore investigated the case where we randomly substituted some Si atoms with $\mathrm{Ge}$ in the nanoribbon crystal. After fully relaxing the structure, we have, however, observed that also this nanoribbon with randomly distributed $\mathrm{Si}$ and $\mathrm{Ge}$ atoms does not work too well as a phonon trap, for essentially the same reason as the perfect modulation: The $\mathrm{Si}$ and Ge randomly distributed supercell is not large enough to confine the low-energy phonon modes. We checked this observation by using a tight-binding approximation to calculate the phonon spectrum. This allows us to reach a larger supercell and thus shows that the phonon thermal conductance decreases due to the phonon confinement in these random structures.

\section{A. Thermoelectric properties of the silicene-germanene nanoribbons}

In this section, we investigate the thermoelectric properties of orderly distributed heterostructured silicene-germanene nanoribbons (SiGeNRs). After forming the structure, we have relaxed the atomic positions, without taking into 
(a) Zigzag-edge

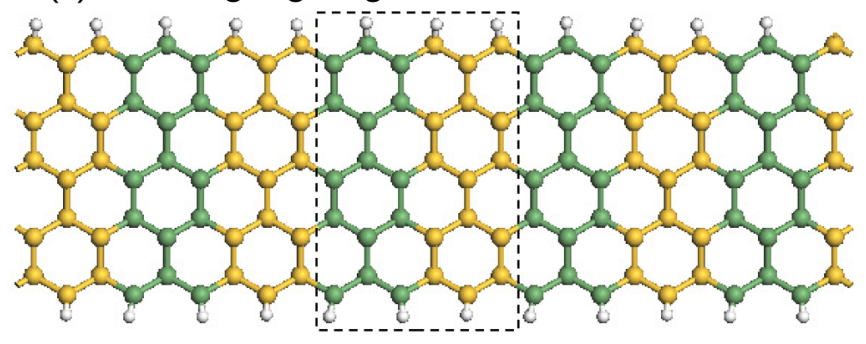

(b) Armchair-edge

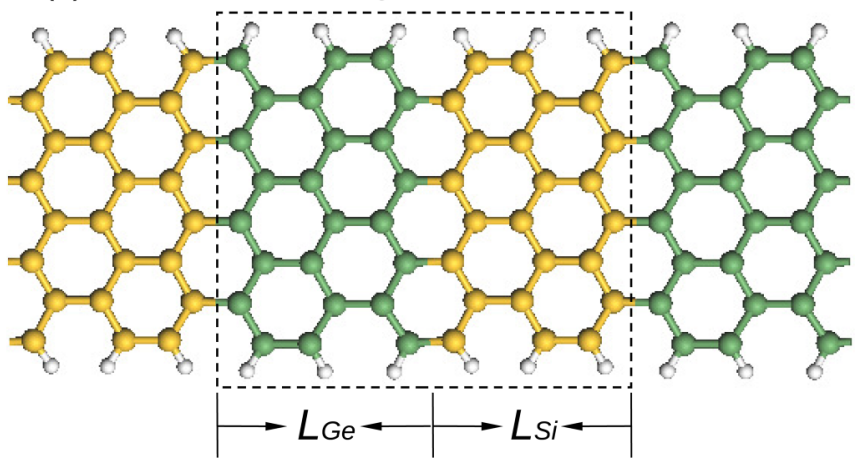

FIG. 11. (Color online) Geometrical structures of (a) ZSiGeNRs and (b) $A$-SiGeNRs, where the line encloses a supercell along the ribbon axis and $L_{\mathrm{Si}}$ and $L_{\mathrm{Ge}}$ are the lengths of silicene and germanene stripes in the supercell, respectively. Here we have chosen $L_{\mathrm{Si}}=L_{\mathrm{Ge}}=3$ and used the hydrogen to passivate the ribbon edges.

account any substrate. Figure 11 shows the optimized geometrical structures of zigzag- and armchair-edged SiGeNRs ( $Z$-SiGeNRs and $A$-SiGeNRs) passivated by hydrogen atoms, where the line encloses a supercell along the ribbon axis. $L_{\mathrm{Si}}$ and $L_{\mathrm{Ge}}$ are the length of silicene and germanene stripes in the supercell, respectively.

We begin with the case $L_{\mathrm{Si}}=L_{\mathrm{Ge}}=1$. In Figs. 12(a) and 12(b) we report the transmission coefficient as a function of electron energy for different widths of the Z-SiGeNRs and $A$-SiGeNRs, respectively. It can be seen that the transmission probability exhibits characteristic quantized steps and a band gap is shown around the Fermi energy. Increasing the ribbon width, the band gap for $Z$-SiGeNRs shows an oscillatory behavior of decreasing amplitude from $W_{Z}=4$ to 7 [see Fig. 12(c)], while the gap for $A$-SiGeNRs shows a strongly oscillatory behavior as shown in Fig. 12(d). When the ribbon width $W_{A}$ satisfies either $3 p$ or $3 p+1$, a larger gap appears than that of the nanoribbons with width $3 p+2$. This width dependence of the band gap is similar to that of the $A$-GeNRs and $A$-SiNRs as we have discussed in Sec. IV. Starting from this transmission function we can now easily evaluate Eqs. (2)(4) to obtain the transport coefficients. In Figs. 12(e) and 12(f) we plot the electrical conductance as a function of the chemical potential $\mu$ in the linear response. It is found that the electric conductance for $Z$-SiGeNRs exhibits a peak and a dip around $\mu=0$. As for the $A$-SiGeNRs, we show that the electrical conductance is zero for the nanoribbon with widths $W_{A}=3 p$ and $3 p+1$ due to the presence of the larger band gap, while the conductance for the ribbon with width $W_{A}=3 p+2$ has a dip at $\mu=0$ where the conductance assumes a finite value. In Figs. 12(g) and 12(h), the Seebeck coefficient versus chemical potential is depicted. It is found that in the Seebeck coefficient, around $\mu=0$ two peaks appear for both $Z$-SiGeNRs and $A$-SiGeNRs with widths $W_{A}=3 p$ and $3 p+1$. The absolute value of the peak for $A$-SiGeNRs is $1.4 \mathrm{mV} / \mathrm{K}$, which is quite a bit larger than the value of the $Z$-SiGeNRs, indicating a quite high thermoelectric effect in this armchair-edged nanoribbon. On the other hand, for the armchair nanoribbons with width $3 p+2$, the Seebeck coefficient is very small due to the very small gap present in these systems. In Figs. 12(i) and $12(\mathrm{j})$ the total thermal conductance $\kappa_{e}+\kappa_{p}$ including electron and phonon contributions is plotted. It can be seen that $\kappa=\kappa_{e}+\kappa_{p}$ for the Z-SiGeNRs exhibits a peak, while for the $A$-SiGeNRs with widths $3 p$ and $3 p+1$, it has a plateau in the energy region around $\mu=0$, mostly due to the
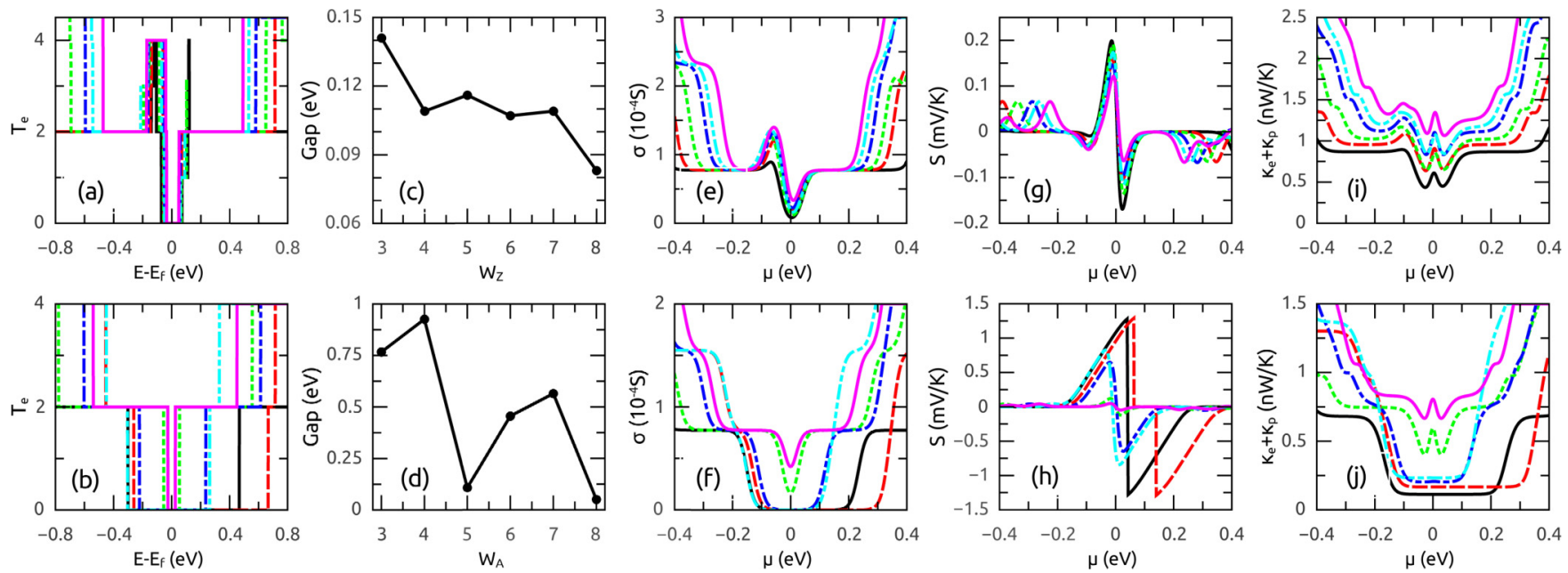

FIG. 12. (Color online) (a), (b) Electron transmission coefficient as a function of energy for Z-SiGeNRs and $A$-SiGeNRs, respectively. (c), (d) Band gap as a function of ribbon widths $W_{Z}$ and $W_{A}$, respectively. (e), (f) Electrical conductance. (g), (h) Seebeck coefficient. (i), (j) Electron and phonon thermal conductances as a function of chemical potential $\mu$ for $Z$-SiGeNRs and $A$-SiGeNRs, respectively, where we have set the temperature $T=300 \mathrm{~K}$. Each curve in (a), (b), and (e)-(j) corresponding to a specified ribbon width endows the same meaning as in Fig. 6. 

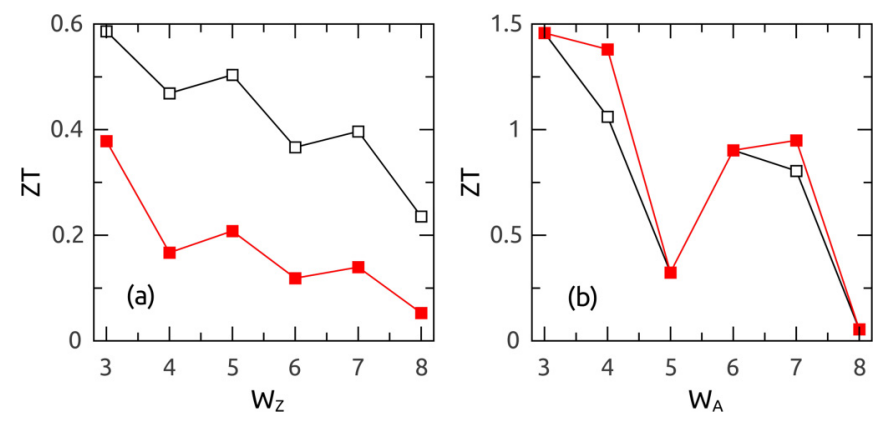

FIG. 13. (Color online) Figure of merit $Z T$ at room temperature for (a) $Z$-SiGeNRs and (b) $A$-SiGeNRs as a function of nanoribbons with widths $W_{Z}$ and $W_{A}$, where the hollow and full points correspond to the hole and electron transport, respectively.

phonon thermal transport. As for the nanoribbon with width $3 p+2$, the thermal conductance reaches a local maximum on account of a local maximum of the electron heat contribution at $\mu=0$.

We find that the thermal conductance of SiGeNRs is between the value of GeNRs and SiNRs, i.e., the thermal conductance of SiGeNRs is larger than that of GeNRs, while smaller than that of SiNRs. Similar to the case of GeNRs or SiNRs, at low temperature region, the linear dependence of the thermal conductance on the temperature is still observed, in agreement with Eq. (10). The behavior at large temperatures is similar to the thermal conductances of GeNRs and SiNRs, therefore we do not report here the complete picture.

In Figs. 13(a) and 13(b), we report the figure of merit $Z T$ of both $Z$-SiGeNRs and $A$-SiGeNRs as a function of ribbon widths $W_{Z}$ and $W_{A}$, respectively. It is found that maximum value of the figure of merit for $Z$-SiGeNRs appears in the narrowest nanoribbon, which is about 0.59 corresponding to the hole transport, while for the electron transport, the corresponding $Z T$ is about 0.38 . As to the armchair-edged nanoribbon with width $W_{A}=3$, the $Z T$ is found to be 1.46 for both the hole and electron transport [see Fig. 13(b)]. With the increase of the ribbon width, the figure of merit shows an oscillatory behavior reminiscent of the different properties of the nanoribbons with different widths. The amplitude of the oscillation, however, decreases quite rapidly with increasing the ribbon width. This is mostly due to the rapid increasing of the phonon thermal conductance with $W_{A}$. In particular, the $Z T$ is very small in the case of the nanoribbon with width $3 p+2$ due to the small Seebeck coefficient as shown in Fig. 12(h).

\section{B. Component modulation of the thermoelectrics in the silicene-germanene nanoribbons}

In Fig. 14, we investigate the thermoelectric properties of SiGeNRs by modulating the component lengths of silicene and germanene stripes in the supercell. In the following, the total length of the supercell is given by $L_{S}=L_{\mathrm{Ge}}+L_{\mathrm{Si}}$. Figures 14(a)-14(f) show the figure of merit $Z T$ at room temperature for the $Z$-SiGeNRs and $A$-SiGeNRs as a function of ribbon width for $L_{\mathrm{Si}}=L_{\mathrm{Ge}}=2,3$, and 4, respectively. It is found that the maximum $Z T$ for hole and electron transport in the case of the $Z$-SiGeNRs is 0.85 and 0.42 for $L_{\mathrm{Si}}=L_{\mathrm{Ge}}=2$, 0.87 and 0.53 for $L_{\mathrm{Si}}=L_{\mathrm{Ge}}=3$, and 1.06 and 0.54 for $L_{\mathrm{Si}}=L_{\mathrm{Ge}}=4$, respectively. For armchair nanoribbons, the maximum of $Z T$ for $L_{\mathrm{Si}}=L_{\mathrm{Ge}}=2$ is about 1.93, while the maximum $Z T$ for $L_{\mathrm{Si}}=L_{\mathrm{Ge}}=3$ or 4 is about 2.18 and 2.06 , respectively. With the increase of the ribbon width, the overall figure of merit decreases for both $Z$-SiGeNRs and $A$-SiGeNRs with widths $W_{A}=3 p$ and $3 p+1$. As to the nanoribbon with width $3 p+2$, the figure of merit is quite small compared to the ribbons with width $3 p$ or $3 p+1$. We found that the
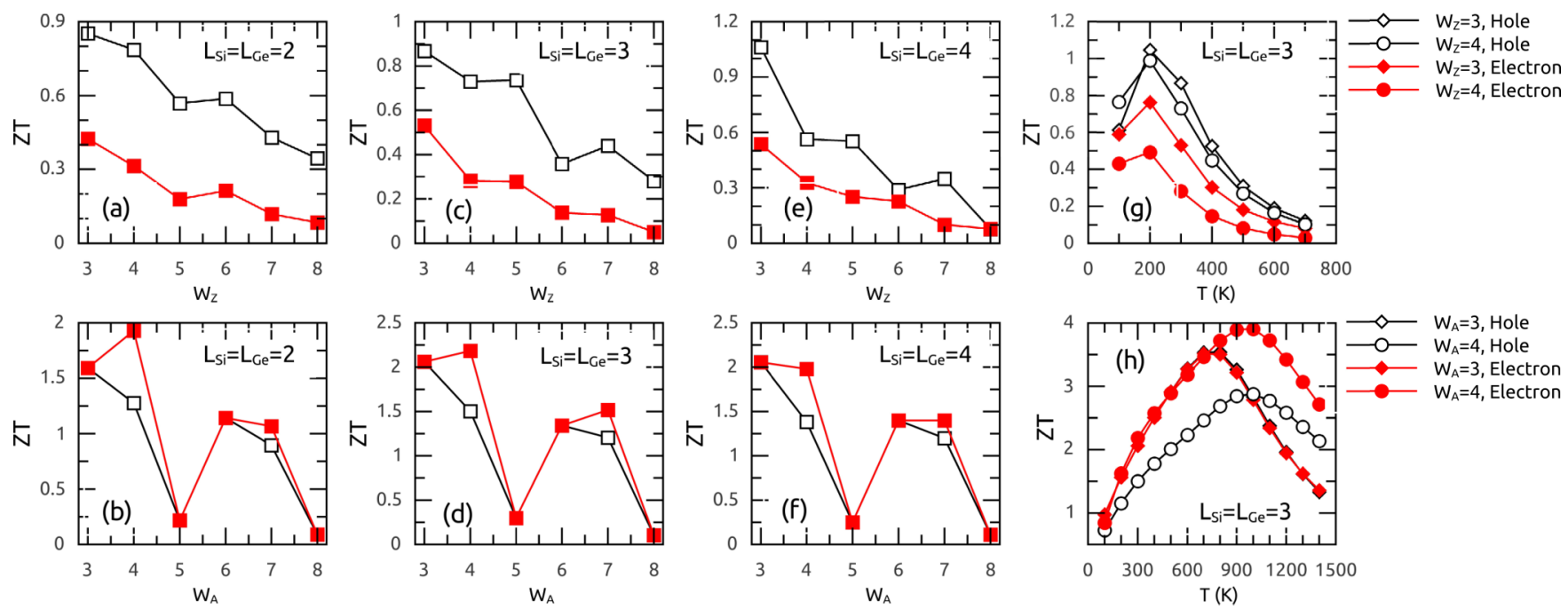

FIG. 14. (Color online) Figure of merit $Z T$ at $T=300 \mathrm{~K}$ for $Z$-SiGeNRs and $A$-SiGeNRs as a function of ribbon widths $W_{Z}$ and $W_{A}$ under different component lengths of silicene and germanene stripes: [(a), (b)] $L_{\mathrm{Si}}=L_{\mathrm{Ge}}=2$, [(c), (d)] $L_{\mathrm{Si}}=L_{\mathrm{Ge}}=3$, and [(e), (f)] $L_{\mathrm{Si}}=L_{\mathrm{Ge}}=4$, respectively. (g), (h) Figure of merit as a function of temperature for Z-SiGeNRs and $A$-SiGeNRs with the corresponding ribbon widths 3 and 4 , where the lengths of the silicene and germanene stripes in the supercell are $L_{\mathrm{Si}}=L_{\mathrm{Ge}}=3$. The hollow and full points correspond to the hole and electron transport, respectively 
Seebeck coefficient for the nanoribbon with width $3 p+2$ is very small due to the small band gap, in agreement with our analysis of the system with $L_{\mathrm{Si}}=L_{\mathrm{Ge}}=1$. Figures $14(\mathrm{~g})$ and 14 (h) show the figure of merit as a function of temperature for the $Z$-SiGeNRs with widths $W_{Z}=3,4$ and the $A$-SiGeNRs with widths $W_{A}=3,4$, respectively. It can be seen that the figure of merit increases and then decreases with increasing the temperature. The maximum $Z T$ for $Z$-SiGeNRs is about 1.05 at $T \approx 200 \mathrm{~K}$, and the maximum $Z T$ for $A$-SiGeNRs is about 3.91 at $T \approx 1000 \mathrm{~K}$.

We wish to point out that the $Z T$ of these systems is larger than that for the pure $A-\mathrm{GeNRs}$ or $A$-SiNRs. This means that nanostructuring can improve the overall energy conversion efficiency. On the other hand, the modest increase in $Z T$ for these nanoribbons shows how this nanostructuring is not effective in blocking the phonon modes. We should reach larger $L_{\mathrm{Si}}$ and $L_{\mathrm{Ge}}$, in order to achieve an efficient trapping of the low-energy phonon modes, as we will discuss briefly at the end of next section.

\section{Disorder effect on the thermoelectrics of silicene-germanene nanoribbons}

In the above discussions, the $\mathrm{Si}$ and $\mathrm{Ge}$ atoms in the nanoribbons are orderly distributed along the growth direction. Here we consider the case in which $\mathrm{Si}$ and $\mathrm{Ge}$ atoms randomly occupy with equal probability the sites of the lattice in Fig. 11. The length of the supercell is $L_{S}=6$ and the number of $\mathrm{Si}$ and $\mathrm{Ge}$ atoms in the supercell are taken the same. Since the armchair nanoribbons show the most promising values of the figure of merit, in Figs. 15(a) and 15(b) we report the figure of merit $Z T$ as a function of the chemical potential $\mu$ for disordered $A$-SiGeNRs with the ribbon widths $W_{A}=3$ and 4 , respectively. As a comparison, we have also plotted the figure of merit for $A$-GeNRs, $A$-SiGRs, and $A$-SiGeNRs. It can be seen that the maximum figure of merit for disordered $A$-SiGeNRs and ordered A-SiGeNRs is nearly twice the value of clean $A$-GeNRs and $A$-SiGRs. The maximum $Z T$ for disordered and ordered $A$-SiGeNRs with width $W_{A}=3$ is about 2 for both electron and hole transport corresponding to the positive and negative chemical potentials, while the maximum $Z T$ for the ribbon width $W_{A}=4$ is 2.18 and 2.56 for electron and 1.5 and 1.8 for hole transport, respectively. The
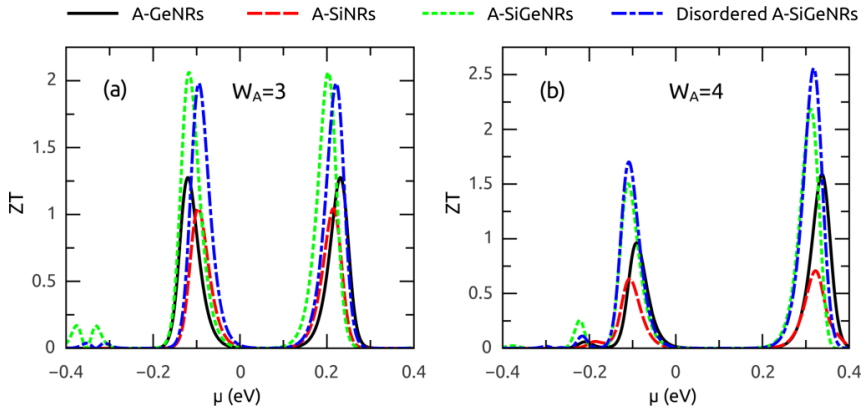

FIG. 15. (Color online) Figure of merit $Z T$ at $T=300 \mathrm{~K}$ for $A$-GeNRs, $A$-SiNRs, $A$-SiGeNRs, and disordered $A$-SiGeNRs with ribbon widths (a) $W_{A}=3$ and (b) $W_{A}=4$ as a function of chemical potential $\mu$, where we have taken the supercell length $L_{S}=L_{\mathrm{Ge}}+$ $L_{\mathrm{Si}}=6$, respectively. principal reason for the enhanced thermoelectric efficiency comes from the reduced phonon thermal conductance, since the electronic properties are slightly affected by the randomness of the atomic positions. Again, due to the small size of the supercell we can consider with $a b$ initio techniques, phonon confinement is not efficient, and therefore the thermal conductance of the disordered and ordered $A$-SiGeNRs is only slightly reduced with respect to the clean $\mathrm{Si}$ or $\mathrm{Ge}$ system as shown in comparing Figs. 14 and 15. For the same reason, the thermal conductance of the random structure is similar to that of the silicene-germanene heterostructures as expected.

To present a proof that a large supercell can effectively further reduce the phonon thermal conductance in the SiGe heterostructures, we use a semiclassical tight-binding method to investigate the lattice thermal transport properties. To obtain the atomic force constant of the system, the Keating potential is used [50-52], which is given by

$$
\begin{aligned}
U= & \frac{1}{2} k_{r} \sum_{i, j}\left(\mathbf{R}_{i, j}^{2}-\mathbf{r}_{i, j}^{2}\right)^{2} \\
& +\frac{1}{2} k_{\theta} \sum_{i, j, k \neq j}\left(\mathbf{R}_{i, j} \cdot \mathbf{R}_{i, k}-\mathbf{r}_{i, j} \cdot \mathbf{r}_{i, k}\right)^{2},
\end{aligned}
$$

where $\mathbf{R}_{i, j}$ and $\mathbf{R}_{i, k}$ are the equilibrium position vectors connecting atom $i$ with $j$ and $k$, and $\mathbf{r}_{i, j}$ and $\mathbf{r}_{i, k}$ are the corresponding position vectors after deformation, respectively. The bond stretching and bending force parameters $k_{r}$ and $k_{\theta}$ for silicene in Eq. (13) are $7.2186 \times 10^{20} \mathrm{~N} / \mathrm{m}^{3}$ and $1.5225 \times 10^{20} \mathrm{~N} / \mathrm{m}^{3}$. These constants can be obtained from the force constants of graphene [52,53]. In our case we have used

$$
k_{r}=\frac{2 \chi}{d}, \quad k_{\theta}=\frac{\varphi}{d},
$$

where $\chi=81 \mathrm{~N} / \mathrm{m}^{2}$ and $\varphi=34 \mathrm{~N} / \mathrm{m}^{2}$ for silicene [52,53] and $d$ is the equilibrium distance of the $\mathrm{Si}$ atoms in the silicene structure, which we have calculated as $d=2.244 \AA$. We have then fine tuned the values of $k_{r}$ and $k_{\theta}$ to improve the agreement between the phonon spectrum (not shown) calculated via $a b$ initio and the one calculated within the tight-binding approximation. For the parameters of germanene, we roughly estimate $k_{r}=5.3469 \times 10^{20} \mathrm{~N} / \mathrm{m}^{3}$ and $k_{\theta}=1.2516 \times$ $10^{20} \mathrm{~N} / \mathrm{m}^{3}$ through comparing the force-constant ratio of this 2D system with the bulk silicon and germanium crystals [50]. We have again fine tuned these values to improve the agreement between the $a b$ initio and tight-binding phonon spectra. For the force parameters between $\mathrm{Si}$ and $\mathrm{Ge}$ atoms in the hybrid structures, we take their average value. As to the $\mathrm{Si}-\mathrm{H}$ and $\mathrm{Ge}-\mathrm{H}$ interactions, we take $10 \%$ of the corresponding $\mathrm{Si}-\mathrm{Si}$ and $\mathrm{Ge}-$ Ge interactions, accordingly. Based on this Keating model and combined with the nonequilibrium Green's function technique, we can calculate the phonon transmission probability and thus the thermal transport properties (see details in Ref. [15]). Figures 16(a) and 16(b) show the phonon thermal conductance calculated from tight binding (gray lines) for $A$-GeNRs, $A$-SiNRs, $A$-SiGeNRs, and disordered $A$-SiGeNRs, where the ribbon width $W_{A}=3$. To check how reliable the tight-binding calculation are, we report together the thermal conductance 


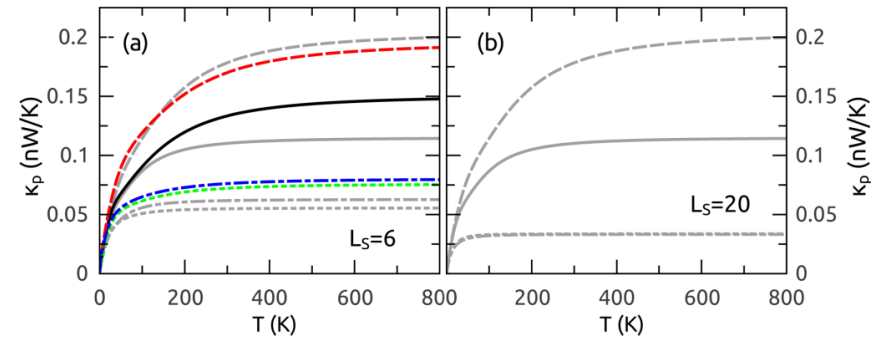

FIG. 16. (Color online) Phonon thermal conductance of various armchair nanoribbons with the ribbon width $W_{A}=3$, where the supercell length $L_{S}$ for $A$-SiGeNRs and disordered $A$-SiGeNRs is (a) $L_{S}=6$ and (b) $L_{S}=20$, respectively. The curves in color are calculated from $a b$ initio and the other curves in gray are calculated from tight binding. The different curves share the same meaning as those in Fig. 15.

calculated from $a b$ initio [color lines in Fig. 16(a)]. It can be seen that the thermal conductance obtained from tight binding and $a b$ initio are quite close, especially in the low temperature region. In addition, it is found that the phonon thermal conductance in the case of $A$-SiGeNRs and disordered $A$-SiGeNRs is drastically decreased compared to the pure $A$-SiNRs and $A$-GeNRs. With further increasing the length of the supercell, the phonon thermal conductance is decreasing as shown in Fig. 16(b).

In Fig. 17, the phonon thermal conductance at $T=$ $300 \mathrm{~K}$ for both $A$-SiGeNRs and disordered $A$-SiGeNRs as a function of supercell length $L_{S}$ is investigated, where $L_{S}$ is defined as the sum of the length of silicene and germanene stripes. It can be seen that the phonon thermal conductance decreases with increasing the length of the supercell, and the $\kappa_{p}$ for both $A$-SiGeNRs and disordered $A$-SiGeNRs are close to each other. This indicates that the larger supercell in the silicene-germanene heterostructures can effectively constrain the phonon transport and that the disordered heterostructure becomes more efficient in confining phonons only at large unit cell lengths. Through checking the transmission probability (not shown), it is found that the weight of the transmission probability is gradually moved to the low-frequency region thus decreasing the total energy flow.
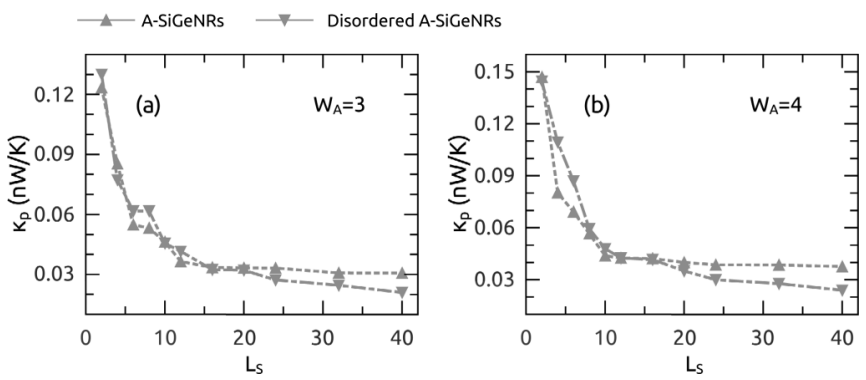

FIG. 17. Phonon thermal conductance $\kappa_{p}$ at $T=300 \mathrm{~K}$ calculated from tight binding as a function of supercell length $L_{S}$ corresponding to the ribbon widths (a) $W_{A}=3$ and (b) $W_{A}=4$, respectively.

\section{CONCLUSIONS}

In summary, we have performed first-principles calculations of the thermoelectric coefficients of both two-dimensional silicene and germanene as well as for $\mathrm{Si}$ and Ge nanoribbons. We have also considered heterostructures of $\mathrm{Si}$ and $\mathrm{Ge}$ stripes to form a nanoribbon, in an attempt to quench the phonon dynamics and thus increase the figure of merit. These systems can be good thermoelectric materials if they can be reliably produced. Also, being based on $\mathrm{Si}$ and $\mathrm{Ge}$, we expect these devices to be easily interfaced with the modern electronic systems, a distinct advantage with respect to other materials which have shown poor integrability with the actual technology.

The figure of merit for thermoelectric energy conversion of $\mathrm{Si}$ and $\mathrm{Ge}$ low-dimensional systems is quite high, in the range of 1 to 2 at room temperature, considering that we have investigated pristine systems where phonons are not confined. For the silicene and germanene systems we have considered both distorted silicene/germanene grown on a silver surface and freestanding silicene/germanene. In these cases we have found the highest figure of merit is about 0.81 for the distorted silicene. It is important to point out that $\mathrm{Si} / \mathrm{Ge}$ nanosheets grown on a $\mathrm{Ag}$ surface show different electrical properties according to the lattice matching: We have considered a $3 \times 3$ $\mathrm{Si}$ lattice on a $4 \times 4 \mathrm{Ag}$ substrate since this induces no stress at the supercell edges and when the Ag substrate is removed, the distorted silicene has a finite band gap. It is indeed clear from our calculations that in order to increase the figure of merit, we need to have a small gap semiconductor since this maximizes the Seebeck coefficient.

Our attempts to quench the phonon dynamics have been hindered by the small scale of the supercell we can calculate with our $a b$ initio techniques. We could in principle go beyond these limitations by using other classical tools such as, e.g., molecular dynamics. However, especially for the nanoribbons, in our tests (not reported here) these tools have proven unable to recover the quantum of thermal conductance at small temperatures. We have therefore chosen to test the phonon confinement with semiempirical techniques, e.g., a tight-binding calculation of the phonon thermal transport for large supercell. We report that the thermal conductance is effectively reduced by about $50 \%$ in going from a supercell made of six units to a supercell made of 20 units.

\section{ACKNOWLEDGMENTS}

We thank Y. Pouillon and A. Iacomino for providing some computational help. We acknowledge financial support from CONSOLIDER INGENIO 2010: NANOTherm (Grant No. CSD2010-00044), Diputacion Foral de Gipuzkoa (Grant No. Q4818001B), the European Research Council Advanced Grant DYNamo (Grant No. ERC-2010-AdG-267374), Spanish Grants (Grant No. FIS2010-21282-C02-01), Grupos Consolidados UPV/EHU del Gobierno Vasco (Grant No. IT578-13), Ikerbasque, and MAT2012-33483. Computational time was granted by i2basque and BSC Red Española de Supercomputacion. 
[1] D. Pollock, Thermoelectricity; Theory, Thermometry, Tool (ASTM, Philadelphia, 1985).

[2] G. S. Nolas, J. Sharp, and H. J. Goldsmid, Thermoelectrics: Basic Principles and New Materials Developments, Springer Series in Material Science Vol. 45 (Springer-Verlag, Berlin, 2001).

[3] H. J. Goldsmid, Springer Series in Material Science, 1st ed. (Springer-Verlag, Berlin, 2010), p. 250.

[4] F. J. DiSalvo, Science 285, 703 (1999).

[5] C. Vining, Nat. Mater. 8, 83 (2009).

[6] R. Venkatasubramanian, E. Siivola, T. Colpitts, and B. O'Quinn, Nature (London) 413, 597 (2001).

[7] N. W. Ashcroft and N. D. Mermin, Solid State Physics (Saunders College Publishing, Philadelphia, 1976).

[8] L. D. Hicks and M. S. Dresselhaus, Phys. Rev. B 47, 12727 (1993).

[9] A. J. Minnich, M. S. Dresselhaus, Z. F. Ren, and G. Chen, Energy Environ. Sci. 2, 466 (2009).

[10] P. Pichanusakorn and P. Bandaru, Mater. Sci. Eng., R 67, 19 (2010).

[11] K. S. Novoselov, A. K. Geim, S. V. Morozov, D. Jiang, Y. Zhang, S. V. Dubonos, I. V. Grigorieva, and A. A. Firsov, Science 306, 666 (2004).

[12] A. Balandin, S. Ghosh, W. Bao, I. Calizo, D. Teweldebrhan, F. Miao, and C. N. Lau, Nano Lett. 8, 902 (2008).

[13] J. H. Seol, I. Jo, A. L. Moore, L. Lindsay, Z. H. Aitken, M. T. Pettes, X. Li, Z. Yao, R. Huang, D. Broido, N. Mingo, R. S. Ruoff, and L. Shi, Science 328, 213 (2010).

[14] R. Prasher, Science 328, 185 (2010).

[15] K. Yang, Y. Chen, R. D'Agosta, Y. Xie, J. Zhong, and A. Rubio, Phys. Rev. B 86, 045425 (2012).

[16] A. I. Hochbaum, R. Chen, R. D. Delgado, W. Liang, E. C. Garnett, M. Najarian, A. Majumdar, and P. Yang, Nature (London) 451, 163 (2008).

[17] A. H. Castro Neto, N. M. R. Peres, K. S. Novoselov, and A. K. Geim, Rev. Mod. Phys. 81, 109 (2009).

[18] N. M. R. Peres, Rev. Mod. Phys. 82, 2673 (2010).

[19] P. Ayala, A. Rubio, and T. Pichler, Rev. Mod. Phys. 82, 1843 (2010).

[20] S. Das Sarma, S. Adam, E. H. Hwang, and E. Rossi, Rev. Mod. Phys. 83, 407 (2011).

[21] V. N. Kotov, B. Uchoa, V. M. Pereira, F. Guinea, and A. H. Castro Neto, Rev. Mod. Phys. 84, 1067 (2012).

[22] S. Cahangirov, M. Topsakal, E. Aktürk, H. Sahin, and S. Ciraci, Phys. Rev. Lett. 102, 236804 (2009).

[23] L. Chen, C.-C. Liu, B. Feng, X. He, P. Cheng, Z. Ding, S. Meng, Y. Yao, and K. Wu, Phys. Rev. Lett. 109, 056804 (2012).

[24] F. Bechstedt, L. Matthes, P. Gori, and O. Pulci, Appl. Phys. Lett. 100, 261906 (2012).

[25] P. De Padova, C. Quaresima, C. Ottaviani, P. M. Sheverdyaeva, P. Moras, C. Carbone, D. Topwal, B. Olivieri, A. Kara, H. Oughaddou, B. Aufray, and G. Le Lay, Appl. Phys. Lett. 96, 261905 (2010).
[26] B. Lalmi, H. Oughaddou, H. Enriquez, A. Kara, S. Vizzini, B. Ealet, and B. Aufray, Appl. Phys. Lett. 97, 223109 (2010).

[27] P. De Padova, O. Kubo, B. Olivieri, C. Quaresima, T. Nakayama, M. Aono, and G. Le Lay, Nano Lett. 12, 5500 (2012).

[28] A. Kara, H. Enriquez, A. P. Seitsonen, L. Lew Yan Voon, S. Vizzini, B. Aufray, and H. Oughaddou, Surf. Sci. Rep. 67, 1 (2012).

[29] B. Feng, Z. Ding, S. Meng, Y. Yao, X. He, P. Cheng, L. Chen, and K. Wu, Nano Lett. 12, 3507 (2012).

[30] H. Enriquez, S. Vizzini, A. Kara, B. Lalmi, and H. Oughaddou, J. Phys.: Condens. Matter 24, 314211 (2012).

[31] H. Jamgotchian, Y. Colignon, N. Hamzaoui, B. Ealet, J. Y. Hoarau, B. Aufray, and J. P. Bibérian, J. Phys.: Condens. Matter 24, 172001 (2012).

[32] P. Vogt, P. De Padova, C. Quaresima, J. Avila, E. Frantzeskakis, M. C. Asensio, A. Resta, B. Ealet, and G. Le Lay, Phys. Rev. Lett. 108, 155501 (2012).

[33] C.-L. Lin, R. Arafune, K. Kawahara, M. Kanno, N. Tsukahara, E. Minamitani, Y. Kim, M. Kawai, and N. Takagi, Phys. Rev. Lett. 110, 076801 (2013).

[34] L. Chen, H. Li, B. Feng, Z. Ding, J. Qiu, P. Cheng, K. Wu, and S. Meng, Phys. Rev. Lett. 110, 085504 (2013).

[35] L. Meng, Y. Wang, L. Zhang, S. Du, R. Wu, L. Li, Y. Zhang, G. Li, H. Zhou, W. A. Hofer, and H.-J. Gao, Nano Lett. 13, 685 (2013).

[36] S. Cahangirov, M. Audiffred, P. Tang, A. Iacomino, W. Duan, G. Merino, and A. Rubio, Phys. Rev. B 88, 035432 (2013).

[37] G. H. Li, A. R. Goni, C. Abraham, K. Syassen, P. V. Santos, A. Cantarero, O. Brandt, and K. Ploog, Phys. Rev. B 50, 1575 (1994).

[38] L. Pan, H. J. Liu, X. J. Tan, H. Y. Lv, J. Shi, X. F. Tang, and G. Zheng, Phys. Chem. Chem. Phys. 14, 13588 (2012).

[39] R. D’Agosta, Phys. Chem. Chem. Phys. 15, 1758 (2013).

[40] R. Landauer, IBM J. Res. Dev. 1, 223 (1957).

[41] H. U. Baranger and A. D. Stone, Phys. Rev. B 40, 8169 (1989).

[42] T. Yamamoto and K. Watanabe, Phys. Rev. Lett. 96, 255503 (2006).

[43] G. Kresse and J. Furthmüller, Phys. Rev. B 54, 11169 (1996).

[44] D. M. Ceperley and B. J. Alder, Phys. Rev. Lett. 45, 566 (1980).

[45] J. P. Perdew and A. Zunger, Phys. Rev. B 23, 5048 (1981).

[46] D. Alfè, Comput. Phys. Commun. 180, 2622 (2009).

[47] G. K. Madsen and D. J. Singh, Comput. Phys. Commun. 175, 67 (2006).

[48] E. H. Hwang and S. Das Sarma, Phys. Rev. B 77, 115449 (2008).

[49] J. Jung, T. Pereg-Barnea, and A. H. MacDonald, Phys. Rev. Lett. 102, 227205 (2009).

[50] H. Rücker and M. Methfessel, Phys. Rev. B 52, 11059 (1995).

[51] J. Wang, Q.-A. Huang, and H. Yu, J. Phys. D: Appl. Phys. 41, 165406 (2008).

[52] S. Y. Davydov, Phys. Solid State 54, 652 (2012).

[53] S. Y. Davydov, Phys. Solid State 52, 184 (2010). 